\title{
Qualidade do sono e depressão: que relações sintomáticas em crianças de idade escolar
}

\author{
Filipa Serrão - Universidade do Minho \\ John Manuel Klein - Universidade de Trier ${ }^{1}$ \\ Alda Gonçalves - Universidade do Minho
}

\begin{abstract}
Resumo
O sono e a depressão têm sido associados, tendo-se questionado se um padrão de sono deficitário era condição necessária e suficiente para o aparecimento de sintomatologia depressiva. Este estudo procura averiguar a prevalência dos problemas de sono e da depressão em crianças em idade escolar. Foram avaliadas em dois momentos 467 crianças da escola primária, com idades compreendidas entre os 6 e os 11 anos de idade $(M=8,70$; $\mathrm{DP}=0,692)$. Os resultados revelam uma diminuição na prevalência ao longo do ano lectivo nos dois quadros nosológicos, verificando-se também uma relação negativa entre o rendimento académico e a sintomatologia depressiva nos dois momentos de avaliação (T1 rho=-0,349; $\mathrm{p}=0,000 ; \mathrm{T} 2$ rho=-0,406; $\mathrm{p}=0,000$ ). Serão discutidas implicação dos resultados para a avaliação e intervenção das crianças, contemplando a possibilidade de uma intervenção precoce de modo a prevenir o aparecimento de um estado depressivo na infância que venha manter ou agravar os problemas do sono.

Palavras-chave: Epidemiologia; Perturbação do sono; Depressão infantil; Rendimento académico.
\end{abstract}

\section{Sleep quality and depression: what symptomatic link in school-aged children}

\begin{abstract}
Sleep and depression have been linked and questioned if a deficit sleep pattern were a sufficient condition for arising of depressive symptoms. This study tries to examine the prevalence of sleep problems and depression in school-aged children. Four-hundred and sixty-seven elementary school-children, aged 6 and 11 years $(M=8,70$; $\mathrm{DP}=0,692)$ were evaluated in two moments. The results revealed that during the school-year both nosological entities reduced prevalence rates, and also a negative association between academic performance and depressive symptoms were found in both evaluation moments ( $\mathrm{T} 1$ rho=-0,349; $\mathrm{p}=0,000 ; \mathrm{T} 2$ rho=-0,406; $\mathrm{p}=0,000$ ). The implications of such data for evaluation and intervention in children will be discussed, taking into account the possibility of a early interventions to prevent onset of depressive states in infancy which maintain and heighten sleep problems.

Keywords: Epidemiology; Sleep disorders; Infant depression; Academic performance.
\end{abstract}

\section{Introdução}

Ao longo dos anos, as pesquisas relacionadas com o sono e com a depressão na infância têm proliferado intensamente, sendo cada vez mais um campo de interesse e de relevância para a comunidade científica. Actualmente, a relação desses quadros nosológicos tem recebido especial atenção dos investigadores pelas implicações que a compreensão dessa relação tem no campo preventivo das desordens emocionais e comportamentais na infância e, por conseguinte, na idade adulta (Riemann, Berger \& Voderholzer, 2001). Por tudo isso, os estudos desenvolvidos neste âmbito primeiramente procuram conhecer as especificidades de cada patologia, para que com este conhecimento seja mais fácil a compreensão da relação que os une (Ford \& Cooper-Patrick, 2001).

Assim, e quanto ao sono, este assume-se como um elemento fundamental para a recuperação física e psicológica do ser humano. É considerado um estado de repouso normal e periódico que se caracteriza especialmente pela suspensão da consciência, pelo relaxamento dos sentidos e dos músculos, pela diminuição do ritmo circulatório e respiratório e pela actividade onírica. Por tudo isso, constitui-se uma necessidade física primordial para uma vida salutar, que permite a restauração física e protege o ser do desgaste natural das horas acordadas (Stores, 2001).

Todavia, e tendo em conta a natureza da vida actual, nem sempre o sono é respeitado, principalmente nas grandes cidades. $\mathrm{O}$ crescente número de actividades e

\footnotetext{
${ }^{1}$ Endereço para correspondência:

Universität Trier, Psychologie - Klinisch Psychologie und Psychotherapie, Fachbereich I

D - 54286 - Trier - Germany

Telefone: ++49651/2012884 - Fax: ++49651/2012886

E-mail: jklein@uni-trier.de
} 
de obrigações a que as crianças estão sujeitas, muitas vezes, podem impor às mesmas, um padrão de sono irregular $\mathrm{e}$ insatisfatório (Busse \& Baldini, 1994). A este nível, a literatura evidencia que a maior parte das crianças, entre os 5 e os 12 anos, dorme cerca de 9 horas e meia por noite, sendo aconselhado um padrão de sono com uma durabilidade superior, cerca de 11 a 12 horas.

Em relação à epidemiologia do sono, os estudos realizados têm demonstrado que aproximadamente um terço das crianças em idade escolar sofre de problemas do sono (Blader, Koplewics, Abikoff \& Foley, 1997; Kahn, e cols., 1989; Rona, Li, Guilford \& Chinun, 1998). Ivanenko, Cabtree e Gozal (2005) corroboraram esses dados assumindo que entre $13 \%$ e $27 \%$ dos pais de crianças entre os 4 e os 12 anos que foram alvos de uma medida de auto-relato indicam que os filhos apresentam problemas de sono, como, por exemplo, resistência na hora de dormir, insónia inicial, sonolência ao acordar, ressonar, despertares nocturnos, sonambulismo e sonolência diurna.

Numa análise mais específica das perturbações de sono, os dados epidemiológicos apontam para uma prevalência de $17 \%$ a $41 \%$ para a insónia e $38 \%$ para as parassónias em crianças com idades compreendidas entre os 3 e os 14 anos (Archbold, Pituch, Panahi \& Chervin, 2002). No nível das parassónias, e segundo o DSM-IV (APA, 2000), entre $10 \%$ e $50 \%$ das crianças de três a cinco anos têm pesadelos de intensidade suficiente para preocupar os pais, enquanto entre $1 \%$ e $6 \%$ das crianças apresentam terrores nocturnos. Ainda de acordo com Archbold e cols. (2002), $10 \%$ e $20 \%$ das crianças têm pelo menos um episódio de sonambulismo, mas a prevalência da perturbação é muito menor, provavelmente entre 1\% e $5 \%$. Quanto às diferenças de género, alguns estudos revelam que há uma maior incidência de problemas do sono, como a insónia, no sexo feminino do que no sexo masculino, para as diferentes faixas etárias (Ford \& Cooper-Patrick, 2001; Sadeh, Raviv \& Gruber, 2000).

Curiosamente, embora tão comuns, os problemas do sono não são com tanta frequência abordados nas consultas pediátricas, muitas vezes porque os pais acham que não são questões médicas e, outras vezes, por não estarem familiarizados com o que seria o comportamento normal em relação ao sono. A este nível, existe uma cota de responsabilidade dos médicos. Nos Estados Unidos realizaram-se alguns estudos em prol da Comissão Nacional de Pesquisas em Distúrbios do Sono, os quais detectaram que os problemas do sono são muito pouco diagnosticados nas consultas médicas, o que seria justificado pela falta de formação nas escolas médicas a este nível (Madeira \& Aquino, 2003).

No que diz respeito à etiologia, subjacentes aos problemas de sono estão factores psicossociais que influenciam determinantemente o sono das crianças. Esses problemas resultam das interacções dinâmicas e das influências bidireccionais entre a criança e o seu ambiente social. Nesse sentido, a vulnerabilidade cultural nas práticas de sono da criança (práticas parentais inadequadas na hora de dormir), a dinâmica familiar (problemas conjugais associados a problemas sexuais ou problemas laborais) e o estresse psicossocial (baixo suporte social e eventos de vida traumáticos como o divórcio e a doença de um dos familiares) assumem um papel central na emergência de problemas do sono na infância (Morrell \& Steele, 2003; Sadeh \& Anders, 1993). Além disso, a psicopatologia materna, como a depressão, ansiedade e distorções cognitivas, está intimamente associada com o padrão disfuncional do sono (Rona e cols., 1998).

Por seu lado, as diferenças individuais, como a saúde da criança (problemas respiratórios), a maturidade desenvolvimental e o próprio temperamento da mesma (irritabilidade), também contribuem para as dificuldades no nível do padrão do sono (Morrell \& Steele, 2003). Ainda nesse sentido, a literatura tem referenciado que as crianças que apresentam um padrão de vinculação inseguro evidenciam elevados níveis de ansiedade, de estresse e de dificuldade em regular emocionalmente situações de separação das figuras de vinculação (Cassidy, 1994; Moran \& Pederson, 1998), o que possivelmente desencadeia distúrbios de sono (Morrell \& Steele, 2003).

Os distúrbios de sono caracterizam-se essencialmente pela dificuldade em iniciar e manter o sono, despertar nocturno, sonambulismo e pela sonolência diurna, que por sua vez estão associados com a diminuição da capacidade de concentração, baixa energia e a lentificação psicomotora (Szuba, 2001). Curiosamente, essa sintomatologia é comum a várias desordens emocionais, como a depressão, o que explica a comorbilidade entre esses quadros nosológicos.

Relativamente à depressão infantil, salienta-se o facto de ter sido há relativamente pouco tempo que esta foi reconhecida como distúrbio mental na infância. Isso porque o conceito de depressão infantil ao longo dos anos tem suscitado alguma controvérsia (Harrington, Kerfoot \& Verduyn, 1999). A psicanálise foi responsável, em larga medida, pela ideia de que não poderia haver depressão na infância, dada a inexistência de um superego. Os seguidores dessa abordagem psicológica argumentavam que a depressão requeria um conjunto de estruturas psicóticas e que o transtorno não surgiria antes do final da adolescência, período mais precoce do seu aparecimento (Rehm \& Sharp, 1996).

Porém, algumas investigações contrariaram esses argumentos. Spitz (1965) mostrou que estados análogos à depressão podem ser observados já em bebé. Contudo, é muito raro isso verificar-se, devendo-se a situações de grande negligência parental. Actualmente, admite-se que

Psico-USF, v. 12, n. 2, p. 257-268, jul./dez. 2007 
depois dos 7 anos a depressão assume contornos clínicos análogos à depressão nos adultos, podendo ser rara antes dessa idade. Deste modo, a presença de depressão em crianças e adolescentes em idade escolar já não é questionável (Rehm \& Sharp, 1996).

Em termos epidemiológicos, na população em geral a prevalência da depressão varia em torno de $4 \%$ a $10 \%$, sendo também visível uma maior incidência entre as mulheres, oscilando de $10 \%$ a $25 \%$, enquanto entre os homens atinge $5 \%$ a $12 \%$ (Zavaschi e cols., 2002). Numa recente revisão sobre a epidemiologia dos distúrbios depressivos, em crianças e adolescentes, Bahls (2002) encontrou o resultado da prevalência-ano para a depressão major de $0,4 \%$ a $3,0 \%$ em crianças e de $3,3 \%$ a $12,4 \%$ em adolescentes. No nível das diferenças de género, Weissman e Klerman (1977) referem que, nas crianças, o sexo feminino tem maior tendência para a depressão que o sexo masculino.

Quanto à definição, não existe consenso acerca da depressão, podendo-se afirmar que se trata de um distúrbio que sofre influência de variáveis biológicas, psicológicas e sociais. A sua manifestação ocorre por meio de sintomas emocionais, como o desânimo, baixa auto-estima e desinteresse em actividades prazerosas; de natureza cognitiva, como pessimismo e desesperança; motivacionais, como apatia e aborrecimento; e ainda sintomas físicos, tais como perda de apetite, dificuldades em dormir e perda de energia (Aglio \& Hutz, 2004; Compas, Eye \& Grant, 1993).

Segundo Mericangaas e Angst (1995), existem inúmeros factores de risco e de protecção para o surgimento da depressão ao longo do desenvolvimento. Assim, algumas características do indivíduo e do seu ambiente parecem potenciar os riscos para a depressão, como o aumento da idade, género feminino, baixo nível socio-económico, traços de personalidade específicos e presença de factores ambientais desencadeadores, como a perda ou afastamento dos pais.

Para além disso, a história familiar de depressão tem sido referenciada como um grande factor de risco para esta desordem. Entre os factores individuais que parecem proteger os indivíduos da depressão estão o sucesso na vida escolar, envolvimento em actividades extracurriculares, hábitos de vida saudáveis, competência social, auto-percepção positiva, competência intelectual, relações sociais positivas e suporte social adequado. Assim, e segundo Steinberg (1999), a maior ou menor probabilidade de surgimento da depressão é vista como o resultado da interacção de uma série de condições ambientais, especialmente estresse, perda e predisposições individuais (Aglio \& Hutz, 2004).

Relativamente aos critérios de diagnóstico, historicamente também tem sido outra área de Psico-USF, v. 12, n. 2, p. 257-268, jul./dez. 2007 discordância no estudo da depressão de crianças e adolescentes (Rehm \& Sharp, 1996). Associado a isso surge a noção de "depressão mascarada", que, segundo Rudolph, Hammen e Burge (1997), deve-se, provavelmente, à observação de quadros clínicos em que a depressão surge como secundária a comportamentos socialmente disruptivos. Ou seja, a depressão surge sob a forma de inúmeros sintomas, que incluem enurese, agressividade, birras, insucesso escolar, isolamento social, medos, problemas de sono, entre outros.

Desse modo, e dada a especificidade e a possibilidade de comorbilidade com as perturbações de comportamento, é possível que os sintomas de externalização sejam mais óbvios, e sejam menosprezados os sintomas depressivos, falando-se assim de "depressão mascarada". A este nível, Strecht (1997) assume que a depressão infantil é feita de múltiplos fragmentos, os equivalentes depressivos, que podem traduzir-se em perturbações funcionais de sono e alimentação, esfincterianas, somáticas, perturbações da aprendizagem escolar e perturbações do comportamento. Clerget (2001) corrobora essa assumpção assumindo que, na faixa etária dos 6-12 anos, o quadro depressivo caracteriza-se por dificuldades escolares e de concentração intelectual, ansiedade, desinteresse, problemas de comportamento, problemas alimentares e de sono.

Por tudo isso, a literatura tem vindo a demonstrar que o sono e a depressão são fenómenos complexos, sendo difícil a compreensão da relação que os une (Ford \& Cooper-Patrick, 2001). Tal como o sono pode ser um sintoma de psicopatologia infantil ou de um significante estressor na vida da criança, a psicopatologia pode resultar do sono insuficiente e da consequente fadiga ou por eles ser exacerbada (Sadeh, Raviv \& Gruber, 2000).

Assim, importa perceber a essência do papel que cada variável assume nesta relação. Por um lado, os esquemas cognitivos presentes na depressão infantil, como a percepção negativa do self, dos outros e do mundo, aumentam o estado da vigília, interferindo assim com o início do sono ou com a manutenção do mesmo, podendo desencadear problemas de sono. Por outro lado, e tendo em conta que a insónia é mais do que um mero processo biológico, a mesma poderá despoletar os determinantes da depressão (Stores, 2003). Isso porque, quando uma pessoa não consegue dormir, pensa que está a perder o controle do seu próprio corpo, o que exacerba sentimentos de desesperança, os quais são fundamentais para o desenvolvimento da depressão.

Nesse sentido, ao longo do tempo, vários estudos interessaram-se pela compreensão da relação na infância e na idade adulta, tendo chegado a resultados significativos. Stein, Mendelsohn, Obermeyer, Amromin e Benca (2001) realizaram um estudo com o 
intuito de avaliar a prevalência dos distúrbios do sono na idade escolar e analisar a associação desses distúrbios com psicopatologia. O que encontraram foi que o aumento da prevalência da depressão, hiperactividade e outras desordens psiquiátricas em crianças e adolescentes pode, de certa forma, ser o resultado de problemas de sono precoces.

A validar essa conjectura surge uma pesquisa levada a cabo por Roberts, Roberts e Chen (2001), que constata inúmeras evidências que um sono com pouca qualidade está associado com problemas emocionais e comportamentais na adolescência. Os estudos realizados nessa faixa etária mostram que o grupo de jovens com problemas emocionais, comportamentais e psiquiátricos apresentam um rácio de distúrbios do sono elevado (Carskadon, 1990; Dahl, 1996; Roberts e cols., 2001). Esta ligação dos distúrbios de sono com a psicopatologia é corroborada ainda por um outro estudo, no qual os adolescentes com problemas do sono apresentam mais problemas de ansiedade, de depressão, irritabilidade, fobias, raiva, instabilidade emocional, comportamento e atenção (Carskadon, 1990; Choquet \& Menke, 1987; Liu e cols., 2000).

Por seu lado, Gillin (1998), numa análise cuidadosa de vários estudos desenvolvidos com o objectivo de entender os predictores de algumas desordens emocionais, verifica que a insónia e a hipersónia são factores de risco para o desenvolvimento posterior ou recorrente da depressão e de estados de ansiedade (Stores, 2003). Weissman, Greenwald, NinoMurcia e Dement (1997) validam esta ideia, ao constatar que a insónia tem sido cada vez mais associada com o aumento do risco de perturbação depressiva major, perturbação de pânico e abuso de álcool. Outros autores, com base nos seus próprios estudos, sugerem que os indivíduos com perturbação do sono apresentam alto risco de desenvolver depressão nos anos posteriores (Ford \& Cooper-Patrick, 2001).

Desse modo, neste momento, o que está claro na comunidade científica é que indivíduos com distúrbios do sono estão em maior risco de desenvolver perturbações de humor e necessitam de acompanhamento. Assim, a sinalização e o tratamento da perturbação do sono antes da ocorrência de um episódio depressivo major assume-se como uma mais-valia no campo preventivo das perturbações de humor em qualquer idade (Ford \& Cooper-Patrick, 2001).

No panorama português não há conhecimento de estudos realizados neste âmbito onde se possa ter uma noção do quadro relacional dessas duas patologias. Desse modo, o estudo em questão surge como forma de avaliar o padrão de prevalência desses dois quadros nosológicos na população infantil, bem como a relação que os une. É de ressalvar que este conhecimento factual irá ser determinante no campo preventivo, já que uma prevalência significativa dessas duas patologias em crianças com idade escolar, associada a uma relação positiva entre esses quadros clínicos, evidencia a necessidade de desenvolver precocemente programas preventivos, visando prevenir o aparecimento de desordens emocionais e comportamentais na infância.

\section{Método}

\section{Participantes}

A amostra do estudo é constituída por 467 alunos do $1^{\circ}$ ciclo do ensino básico, que frequentam escolas do concelho de Braga, 253 (54,18\%) do sexo feminino e 214 $(45,82 \%)$ do sexo masculino, com idades compreendidas entre os 6 e os 11 anos de idade $(M=8,70 ; D P=0,692)$. $A$ maioria $(58,8 \%)$ dos participantes frequenta $\mathrm{o} 4^{\circ}$ ano de escolaridade e pertence ao nível sócio-económico médio alto $(51,9 \%)$.

\section{Instrumentos}

Todos os participantes deste estudo foram alvos de um questionário composto por três partes: uma ficha sócio-demográfica que visa recolher informação sobre dados biográficos, sociais e académicos; um questionário de experiências de sono-vigília com o fim de aceder informações acerca do padrão de sono e um inventário de depressão infantil com o intuito de avaliar a sintomatologia depressiva.

\section{Ficha sócio-demográfica da criança}

Esta ficha tem como objectivo central possibilitar o acesso a dados pessoais e a características sóciodemográficas dos participantes, de modo a permitir a caracterização da amostra no que se refere a idade, sexo, nacionalidade, nível sócio-económico, ano de escolaridade e rendimento académico.

\section{Questionário de Experiências de Sono-Vigilia (V an Diest, 1999)}

Foi usada a versão portuguesa desta escala (Klein, Fernandes \& Gonçalves, 2005a), a qual revelou boas características psicométricas. Este questionário é uma medida padronizada da qualidade do sono e da vigília, muito usada na investigação epidemiológica. $\mathrm{O}$ questionário possui 14 itens distribuído por 9 dimensões, correspondendo a 9 tipos de queixas somáticas da perturbação de sono, em que a resposta é dada segundo uma escala tipo Likert. Para além disso, o questionário tem duas versões, uma para crianças e outra para adultos.

A distribuição dos 14 itens pelas 9 dimensões é feita do seguinte modo: (Ii) insónia inicial: itens 1 e 2; (Im) insónia de manutenção: itens 3 e 4; (It) insónia terminal: 
itens 5 e 6; (Sa) sonolência ao acordar: itens 7 e 8; (Ca) cansaço ao acordar: itens 9 e 10; (Sd) sonolência diurna: item 11; (Rs) ressonar: item 12; (St) sestas: item 13 e (Ds) duração diária do sono: item 14. Quanto à cotação dos itens, vai de 1 a 4 , correspondendo o valor " 1 " às categorias "de modo nenhum" e "nunca", e o valor "4" às categorias "muito grave" e "sempre".

Relativamente às queixas Ii, Im, It, Sa e Ca, para cada uma há 2 itens: um item específico (s) que permite avaliar a presença de determinada queixa e um item de frequência de ocorrência (f). Por exemplo, para a queixa "insónia inicial" (Ii), o item 1 permite avaliar se o indivíduo se queixa de insónia inicial e o item 2 permite avaliar a frequência com que essa queixa ocorre (a sua intensidade). Assim, para estas queixas temos de determinar o produto dos 2 itens. Por exemplo, supondo que um indivíduo respondeu ao item " 1 " colocando a cruz em "Um problema grave" e ao item " 2 " colocando a cruz em "Poucas vezes", neste caso, a cotação para a queixa Ii é 3 × $2=6$.

A soma dos valores de Ii, Im, It, Sa, Ca e $\mathrm{Sd}$ permite obter o valor total de perturbação do sono. Nesse sentido, para as diferentes queixas, pode obter-se o escore máximo de 16 (4 x 4) e o escore mínimo de 4 (4 x 1), que possibilita categorização clínica das queixas em perturbação leve (4 a 8), perturbação moderada (8 a 12) e perturbação grave (12 a 16). Além disso, com base nos escores totais das queixas, é possível estratificar a perturbação do sono em perturbação leve $(27>$ total $<35)$, perturbação moderada $(35>$ total $<42)$ e perturbação grave (total $>42)$. O valor de perturbação subjectivamente avaliado pelo QESV varia num intervalo de 6 a 81. Por outro lado, os itens 12 e 13 avaliam aspectos que podem interferir com o sono nocturno (ressonar e sestas) ou sugerir sonolência diurna (sestas). O item 14 permite avaliar a duração do sono por dia.

Children's Depression Inventory (CDI; Kovacs, 1985, traduzido e validado por Gonçalves \& Dias, 1999)

O CDI é um inventário que visa detectar a presença e a severidade da desordem depressiva na infância. Trata-se de um instrumento de auto-relato de 27 itens desenvolvido para avaliar a intensidade das queixas depressivas em crianças e adolescentes em idades compreendidas entre os 7 e os 17 anos. Cada item do CDI é composto por três frases, classificadas com um valor de 0,1 e 2, com os valores mais elevados significando uma maior severidade dos sintomas. Quanto ao valor de corte, este está dividido por dois grupos etários. O grupo etário entre os 8 e os 12 anos tem como ponto de corte o escore 16, sendo considerado um valor clínico quando o escore total é igual ou superior a 16. O grupo etário entre os 13 e os 17 anos tem como ponto de corte o escore 20, pelo que valores iguais ou acima deste reflectem patologia.

Psico-USF, v. 12, n. 2, p. 257-268, jul./ dez. 2007

\section{Procedimentos}

No que concerne ao procedimento, o estudo foi realizado ao longo do ano lectivo de 2005/2006 em dois momentos de avaliação, um primeiro momento em dezembro (T1) e um segundo momento em abril (T2). Primeiramente foram estabelecidos contactos com as escolas e professores para a elaboração de um plano de recolha de dados, o qual não interferisse nas actividades escolares e no bom funcionamento da sala de aula. Além disso, e porque se tratava de menores, procedeu-se à elaboração de um pedido de autorização aos agentes educativos, no qual constava o tema da investigação e os objectivos correspondentes, bem como a importância e a utilidade da mesma, de forma a obter o consentimento informado dos participantes. Seguidamente, foram aplicados os questionários aos alunos por intermédio dos professores, sendo este um requisito que constava no plano inicialmente acordado com a direcção da escola, para não pôr em causa o bom funcionamento das turmas.

Neste sentido, no T1, a amostra foi recolhida em 11 escolas do concelho de Braga, obtendo-se uma amostra total de 544 crianças em idade escolar. Porém, uma das escolas de Braga, após facultada a autorização, não permitiu a identificação dos alunos, o que levou à sua exclusão da amostra (morte experimental de 14,15\%). Quanto ao T2, este foi delineado segundo os mesmos parâmetros do T1 junto das restantes escolas, obtendo-se uma amostra de 475 participantes, dos quais 8 foram excluídos por não preencherem o questionário, pelo que se obteve uma amostra final de 467 participantes. É de salientar ainda que, na selecção da amostra, considerou-se como únicos critérios o facto dos participantes frequentarem o $3^{\circ}$ e $4^{\circ}$ ano de escolaridade e procederem à sua identificação nos questionários, para assim, poderem ser participantes válidos no segundo momento de avaliação.

\section{Resultados}

Para aferir o significado dos resultados obtidos, inicialmente irão ser apresentadas as estatísticas descritivas do índice de perturbação de sono e do índice de perturbação de humor. De seguida, proceder-se-á à análise das estatísticas inferenciais que permitirão inferir algumas ilações acerca da relação que une o padrão de sono e a desordem depressiva na infância, bem como a relação dessas duas variáveis com o rendimento académico. Essa análise e tratamento estatístico dos dados foi realizada com o recurso a diversos procedimentos, descritos de seguida, utilizando o programa estatístico Statistical Package for Social Sciences (SPSS - versão 11.5 para Windows). É de ressalvar que, após a verificação se a amostra tinha uma distribuição normativa ou não, pôde-se concluir que a amostra é 
não-normativa (T1 Simetria $=1,780 \quad(\mathrm{EP}=0,191) \quad \mathrm{e}$ Achatamento =5,361 (EP=0,379); $(\mathrm{T} 2$ Simetria $=1,605$ $(\mathrm{EP}=0,198)$; Achatamento=4,506 $(\mathrm{EP}=0,394)$, pelo que se utilizaram testes estatísticos não-paramétricos.

\section{Duração do sono da amostra}

Recorrendo à Figura 1 é possível verificar que, no primeiro momento de avaliação (T1), 44,9\% das crianças dormem mais de 9 horas, 26,2\% dormem menos de 9 horas e uma percentagem menor $(14,4 \%)$ dorme menos de 5 horas. Porém, no segundo momento de avaliação (T2), constata-se um aumento do número de crianças que dormem 9 ou mais horas $(56,0 \%)$, e consequentemente uma diminuição das que dormem menos de 9 horas $(14,0 \%)$ e menos de 5 horas $(12,0 \%)$.

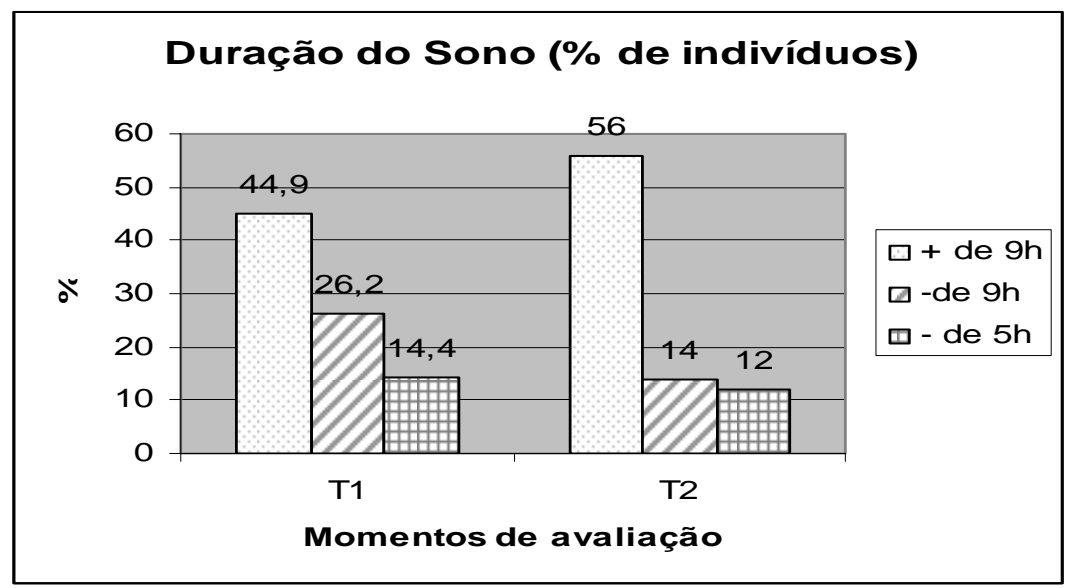

Figura 1 - Duração do sono nos dois momentos de avaliação

Duração do sono, qualidade do sono e género

No que concerne ao T1, os resultados do teste Mann-Whitney para amostras independentes não revelam diferenças significativas entre o sexo e a duração de sono, pelo que se infere que os sexos não se distinguem relativamente à duração de sono (U Mann-Whitney: $Z=$ $-0,604 ; p=0,546)$. Por outro lado, para avaliar a relação entre a duração do sono e qualidade do sono, utilizou-se a correlação de Spearman. Nesse sentido, verifica-se uma relação marginal entre a duração e a qualidade do sono (rho=0,294; $\mathrm{p}=0,000)$. Quanto ao T2, observam-se resultados semelhantes ao $\mathrm{T} 1$, em que os sexos não se distinguem quanto à duração de sono (U Mann-Whitney: $Z=-0,270 ; p=0,787)$, bem como constata-se uma relação, embora pequena, entre a duração do sono e a qualidade de sono $(\mathrm{rho}=0,225 ; \mathrm{p}=0,006)$.

Número de casos sinalizados em termos de escore total e queixas sintomáticas

A este nível, importa primeiramente esclarecer o intervalo de variação do índice de perturbação de sono. Assim, no T1, o intervalo variou entre 7 e 52, com uma média de 17,91 e um desvio padrão de 6,48, enquanto no T2 o intervalo variou entre 9 e 45, com uma média de 17,54 e um desvio padrão 5,38. Relativamente ao índice de perturbação de sono, constatou-se que em T1 92\% dos casos apresenta ausência de perturbação e $8 \%$ apresenta perturbação do sono, sendo essa percentagem distribuída por perturbação leve $(6,8 \%)$ e perturbação grave $(1,2 \%)$. Todavia, em T2 denota-se uma diminuição dos casos clínicos, havendo apenas $3,4 \%$ de casos com perturbação, nomeadamente perturbação leve $(2,0 \%)$, perturbação moderada $(0,7 \%)$ e perturbação grave $(0,7 \%)$.

Quanto à análise das queixas sintomáticas do sono, na Tabela 1 é possível analisar a distribuição das frequências dos resultados obtidos a esse nível nos dois momentos de avaliação; assim, no T1 os resultados obtidos apontam para: insónia inicial (14,4\% perturbação leve - 0,5\% perturbação grave); insónia de manutenção $(17,6 \%$ perturbação leve - 1,6\% perturbação moderada - 1,1\% perturbação grave); insónia terminal (9,1\% perturbação leve); sonolência ao acordar (7,0\% perturbação leve - 3,7\% perturbação moderada - 1,1\% perturbação grave); cansaço ao acordar $(6,4 \%$ perturbação leve $-1,6 \%$ perturbação moderada); sonolência diurna (5,3\% perturbação leve $4,3 \%$ perturbação moderada; $24,6 \%$ - perturbação grave); ressonar (19,8\% perturbação leve - 3,2\% perturbação moderada - 3,2\% perturbação grave) e sestas $(8,0 \%$ perturbação leve $-2,7 \%$ perturbação moderada $-9,1 \%$ perturbação grave). 
Tabela 1 - Análise das queixas sintomáticas do sono

\begin{tabular}{|c|c|c|c|c|c|c|c|c|c|c|c|c|c|c|c|c|}
\hline \multirow{3}{*}{$\begin{array}{c}\text { Queixas } \\
\text { sintomáticas }\end{array}$} & \multicolumn{8}{|c|}{$1^{\circ}$ Momento $(\mathrm{N}=467)$} & \multicolumn{8}{|c|}{$2^{\circ}$ Momento $(\mathrm{N}=467)$} \\
\hline & \multicolumn{2}{|c|}{$\begin{array}{c}\text { Ausência } \\
\text { perturbação }\end{array}$} & \multicolumn{2}{|c|}{$\begin{array}{c}\text { Perturbação } \\
\text { leve }\end{array}$} & \multicolumn{2}{|c|}{$\begin{array}{c}\text { Perturbação } \\
\text { moderada }\end{array}$} & \multicolumn{2}{|c|}{$\begin{array}{l}\text { Perturbação } \\
\text { grave }\end{array}$} & \multicolumn{2}{|c|}{$\begin{array}{c}\text { Ausência } \\
\text { perturbação }\end{array}$} & \multicolumn{2}{|c|}{$\begin{array}{c}\text { Perturbação } \\
\text { leve }\end{array}$} & \multicolumn{2}{|c|}{$\begin{array}{c}\text { Perturbação } \\
\text { moderada }\end{array}$} & \multicolumn{2}{|c|}{$\begin{array}{c}\text { Perturbaçãa } \\
\text { grave }\end{array}$} \\
\hline & $\mathrm{N}$ & $\%$ & $\mathrm{~N}$ & $\%$ & $\mathrm{~N}$ & $\%$ & $\mathrm{~N}$ & $\%$ & $\mathrm{~N}$ & $\%$ & $\mathrm{~N}$ & $\%$ & $\mathrm{~N}$ & $\%$ & $\mathrm{~N}$ & $\%$ \\
\hline Insónia inicial & 397 & 85 & 67 & 14,4 & 0 & 0 & 2 & 0,5 & 337 & 72,2 & 37 & 8 & 0 & 0 & 0 & 0 \\
\hline $\begin{array}{l}\text { Insónia de } \\
\text { manutenção }\end{array}$ & 92 & 19,7 & 82 & 17,6 & 7 & 1,6 & 5 & 1,1 & 315 & 67,4 & 55 & 11,8 & 5 & 1,1 & 0 & 0 \\
\hline Insónia terminal & 425 & 90,9 & 42 & 9,1 & 0 & 0 & 0 & 0 & 327 & 70,1 & 40 & 8,6 & 7 & 1,6 & 0 & 0 \\
\hline Sonolência ao acordar & 412 & 88,2 & 33 & 7,0 & 17 & 3,7 & 5 & 1,1 & 319 & 68,4 & 33 & 7,0 & 17 & 3,7 & 5 & 1,1 \\
\hline Cansaço ao acordar & 430 & 92,0 & 30 & 6,4 & 7 & 1,6 & 0 & 0 & 360 & 77,0 & 7 & 1,6 & 5 & 1,1 & 2 & 0,5 \\
\hline Sonolência diurna & 307 & 65,8 & 25 & 5,3 & 20 & 4,3 & 115 & 24,6 & 290 & 62,0 & 35 & 7,5 & 25 & 5,3 & 25 & 5,3 \\
\hline Ressonar & 345 & 73,8 & 92 & 19,8 & 15 & 3,2 & 15 & 3,2 & 319 & 68,4 & 42 & 9,1 & 7 & 1,6 & 5 & 1,1 \\
\hline Sestas & 375 & 80,2 & 37 & 8,0 & 13 & 2,7 & 42 & 9,1 & 304 & 65,2 & 37 & 8,0 & 15 & 3,2 & 17 & 3,7 \\
\hline
\end{tabular}

Relativamente ao $\mathrm{T} 2$, os dados apresentados permitem constatar uma redução das queixas sintomáticas do sono, excepto da queixa sonolência ao acordar, na qual há um aumento ao longo do período avaliativo.

\section{Perturbação do sono e género}

Em termos de diferenças de género, e com base na Figura 2, é possível constatar que existem diferenças entre os géneros no nível da perturbação de sono, tanto para o T1 como para o T2. Assim, no T1 os rapazes apresentam valores superiores $(\mathrm{M}=19,12$; $\mathrm{DP}=7,81)$ do que as raparigas $(\mathrm{M}=16,78 ; \mathrm{DP}=4,72)$, verificando-se uma diferença estatisticamente significativa [ $\mathrm{t}(124,767)=2,276 ; \mathrm{p}=0,25]$. Todavia, as diferenças entre esses dois grupos não são clinicamente significativas, na medida em que apenas explicam $1,3 \%$ da variância, sendo assim um efeito pequeno. Quanto ao T2, os resultados são semelhantes ao momento anterior, havendo novamente valores de perturbação do sono superiores nos rapazes $(\mathrm{M}=18,95 ; \mathrm{DP}=6,26)$ do que nas raparigas $(\mathrm{M}=16,39$; $\mathrm{DP}=4,25)$, verificando-se uma diferença estatisticamente significativa entre os géneros $[\mathrm{t}(111,117)=$ 2,852; $\mathrm{p}=0,005)]$. No entanto, essa diferença também não é clinicamente significativa, porque apenas explica $5,2 \%$ da variância, o que é um efeito muito pequeno.

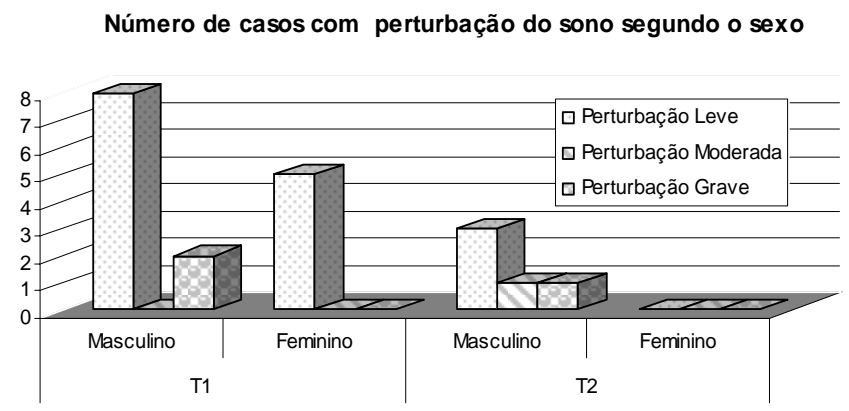

Figura 2 - Número de casos com perturbação do sono, segundo o sexo, nos dois momentos de avaliação 
Sintomas-chave do sono e respectivas diferenças ao longo dos dois momentos de avaliação

Recorrendo a uma análise comparativa dos dois momentos de avaliação, constatam-se diferenças estatisticamente significativas em duas queixas sintomáticas do sono, nomeadamente sonolência ao acordar e sonolência diurna (cf. Tabela 2). Assim, para a dimensão sonolência ao acordar existe uma diferença estatisticamente significativa entre T1 e T2 (Wilcoxon
Signed Rank Test: $Z=-2,229 ; \quad p=0,021)$, que corresponde a um aumento dessa queixa sintomática ao longo do período avaliativo (média T1=2,75 e média $\mathrm{T} 2=3,14)$. Relativamente à sonolência diurna, constatase também uma diferença estatisticamente significativa entre os dois momentos (Wilcoxon Signed Rank Test: $Z=-4,482 ; \quad \mathrm{p}=0,000) ;$ no entanto, essa diferença corresponde a uma diminuição da queixa sintomática (média $\mathrm{T} 1=1,88$ e média T2=1,43).

Tabela 2 - Queixas sintomáticas e resultados do teste Wilcoxon referentes aos dois momentos de avaliação

\begin{tabular}{lcccccc}
\hline \multicolumn{1}{c}{ Queixas sintomáticas } & M & T1 & DP & T2 & DP & Z \\
\hline Insónia inicial & 3,88 & 1,32 & 3,77 & 1,08 & $-0,315$ & 0,753 \\
Insónia de manutenção & 4,30 & 2,03 & 4,10 & 1,36 & $-0,842$ & 0,400 \\
Insónia terminal & 2,79 & 1,58 & 2,98 & 1,96 & $-1,133$ & 0,257 \\
Sonolência ao acordar & 2,75 & 2,41 & 3,14 & 2,69 & $-2,299$ & 0,021 \\
Cansaço ao acordar & 2,23 & 1,82 & 2,10 & 1,88 & $-1,471$ & 0,141 \\
Sonolência diurna & 1,87 & 1,29 & 1,42 & 0,88 & $-4,482$ & $<0,000$ \\
Ressonar & 1,35 & 0,69 & 1,19 & 0,52 & $-2,011$ & 0,044 \\
Sestas & 1,40 & 0,91 & 1,32 & 0,76 & $-1,213$ & 0,225 \\
Duração do sono & 4,62 & 1,78 & 4,83 & 1,70 & $-2,537$ & 0,011 \\
\hline
\end{tabular}

\section{Sintomatologia depressiva}

No que diz respeito à presença de sintomatologia depressiva, no T1 observa-se 6,2\% de casos com sintomatologia clinicamente significativa $(\mathrm{M}=8,55 ; \mathrm{DP}=4,57)$, enquanto no $\mathrm{T} 2$ o número de casos diminui para 4.0\% $(\mathrm{M}=7,79 ; \mathrm{DP}=4,42)$. Por outro lado, procedendo à análise comparativa entre T1 e T2, não se verificam alterações estatisticamente significativas (Wilcoxon Signed Rank Test: MédiaT1=8,39 e Média T2=7,93; $Z=-1,851$; $\mathrm{p}=0,064)$. Importa também salientar, que não se encontram diferenças estatisticamente significativas em relação ao género, idade, nível sócio-económico e rendimento académico.

\section{Depressão e perturbação do sono}

Por outro lado, para analisar a associação entre depressão infantil e perturbação de sono procedeu-se ao cálculo da correlação (rho de Spearman) entre essas duas variáveis. Os resultados apresentados na Tabela 3 mostram uma associação positiva e significativa entre a perturbação de sono e a perturbação de humor em T1 (rho=0,236; $\mathrm{p}=0,001)$ e em T2 (rho=0,220; $\mathrm{p}=0,007)$.

Tabela 3 - Análise estatística da associação dos problemas do sono com a sintomatologia depressiva $(\mathrm{N}=467)$.

\begin{tabular}{ccccccccccc}
\hline & P Sono & II & IM & IT & SA & CA & SD & RS & ST & DS \\
\hline $\begin{array}{c}\text { CDI T1 } \\
\begin{array}{c}\text { Spearman rho } \\
\text { CDI T2 }\end{array}\end{array}$ & $0,236^{* *}$ & $-0,046$ & 0,133 & $0,168^{*}$ & 0,140 & $0,216^{* *}$ & $0,242^{* *}$ & $-0,030$ & 0,197 & $-0,127$ \\
Spearman rho & $0,220^{* *}$ & $-0,134$ & $-0,170^{*}$ & $0,179^{*}$ & $0,198^{*}$ & $0,281^{* *}$ & $0,293^{* *}$ & 0,136 & 0,127 & $-0,138$ \\
\hline
\end{tabular}

$* \mathrm{p}<0,005 ; * * \mathrm{p}<0,001$

Além disso, e de forma a compreender essa associação positiva, recorreu-se a uma análise mais específica por meio do cálculo da correlação entre as queixas sintomáticas da perturbação do sono e os índices de depressão. Assim, é possível observar uma relação significativa entre a depressão e algumas queixas sintomáticas nos dois momentos avaliativos (cf. Tabela 3). Nesse sentido, no T1 constata-se uma associação positiva com a insónia terminal ( $\mathrm{rho}=0,168 ; \mathrm{p}=0,022)$, cansaço ao acordar (rho=0,216; $\mathrm{p}=0,03)$ e com a sonolência diurna 
(rho=0,242; $\mathrm{p}=0,001)$, enquanto no $\mathrm{T} 2$ se verifica uma relação positiva com a insónia terminal (rho $=0,179$; $\mathrm{p}=0,029)$, sonolência ao acordar (rho=0,198; $\mathrm{p}=0,015)$, cansaço ao acordar (rho $=0,281 ; \mathrm{p}=0,000)$ e com a sonolência diurna $(\mathrm{rho}=0,293 ; \mathrm{p}=0,000)$.

\section{Rendimento académico, perturbação do sono e depressão}

Em relação ao rendimento académico não se verificam alterações (média T1=1,62 e média T2=1,52) estatisticamente significativas ao longo do período de avaliação (Wilcoxon Signed Rank Test: $Z=-1,693$; $\mathrm{p}=0,091)$. Por outro lado, ao relacionar o rendimento académico com o escore total da perturbação do sono não se obtém nenhuma correlação significativa. No entanto, ao extrapolar essas correlações para o âmbito das queixas sintomáticas do sono, encontra-se uma associação negativa entre o rendimento académico e a sonolência diurna, nos dois momentos de avaliação ( $T 1$ rho=-0,189; $\mathrm{p}=0,020 ; \mathrm{T} 2$ rho=-0,162; $\mathrm{p}=0,027)$. Para além disso, no T2 obteve-se uma associação positiva entre a duração de sono e o rendimento académico ( $\mathrm{rho}=0,174 ; \mathrm{p}=0,033)$. Ao analisar a associação entre rendimento académico e depressão infantil, os resultados apontam para uma relação negativa e estatisticamente significativa entre essas duas variáveis (T1 rho=-0,349; $\mathrm{p}=0,000 ; \mathrm{T} 2$ rho=-0,406; $\mathrm{p}=0,000)$.

\section{Discussão}

Com esta investigação pretendeu-se averiguar a prevalência do padrão de sono e da sintomatologia depressiva em crianças com idade escolar, bem como avaliar a relação existente entre esses dois quadros nosológicos. No que diz respeito ao padrão de sono, os resultados do estudo aqui apresentado vão ao encontro da literatura existente (Busse \& Baldini, 1994; Klein e cols., 2005b), a qual refere que a maior parte das crianças entre os 5 e os 12 anos dorme cerca de 9 horas e meia por noite. Além disso, os dados mostram que os rapazes e as raparigas não se distinguem quanto à duração do sono.

Estes dados não confirmam os resultados encontrados em alguns estudos (Gau \& Soong, 1995; Sadeh e cols., 2000). O estudo desenvolvido por Gau e Song (1995) sugere que os rapazes dormem mais do que as raparigas. Porém, Sadeh e cols. (2000) apresentam resultados contraditórios, assumindo que as raparigas dormem mais do que os rapazes. Por tudo isso, torna-se claro que a questão das diferenças de género é muito complexa, pelo que a própria literatura não esclarece devidamente esta assumpção. Por outro lado, nesta amostra verifica-se uma relação, embora de pouca magnitude, entre a duração sono e a qualidade de sono, podendo assim inferir-se que a durabilidade do sono tem impacto na qualidade do sono subjectivamente avaliada

Psico-USF, v. 12, n. 2, p. 257-268, jul./dez. 2007 pelos participantes. Todavia, essa relação marginal indica a possibilidade da presença de outros factores mediadores, sendo importante estudos posteriores averiguarem essa associação.

Relativamente à perturbação de sono, no primeiro momento de avaliação verifica-se que $8 \%$ da amostra apresenta sintomatologia de sono, sendo essa percentagem inferior no segundo momento. Esses dados aproximam-se dos resultados obtidos no estudo realizado por Stein e cols. (2001), que encontrou uma prevalência de 10\% para problemas de sono na idade escolar, sendo esta obtida por meio do auto-relato dos pais. No entanto, uma prevalência superior foi encontrada em outros estudos epidemiológicos (Ivanenko, Cabtree \& Gozal, 2005; Sadeh e cols., 2000). Ivanenko e cols. (2005), advogam que aproximadamente 13 a $27 \%$ dos pais de crianças-alvo de uma medida de auto-relato reconhecem que os seus filhos sofrem de problemas de sono. Uma percentagem semelhante, $18 \%$, foi encontrada num estudo levado a cabo por Sadeh e cols. (2000). Um outro dado obtido neste estudo revela que em ambos os momentos de avaliação o número de rapazes com perturbação do sono é ligeiramente superior ao das raparigas. Esse dado não vai ao encontro da literatura encontrada, a qual assume que o sexo feminino tem tendência a apresentar mais problemas de sono do que o sexo masculino, sendo válido para as diferentes faixas etárias (Ford \& Cooper-Patrick, 2001). A par disso, foi ainda possível verificar que o índice de perturbação do sono diminuiu ao longo do ano lectivo. Essa alteração poderá estar relacionada com o facto de, ao longo do período escolar, o padrão de sono ter sido estruturado. De facto, o contexto escolar assume-se como um contexto extremamente estruturante pelas suas rotinas e regras e, principalmente, pelos seus horários, o que, por conseguinte, tem impacto, ao nível da higiene de sono das crianças (Gozal, 1998).

Assim, pode-se inferir que, à medida que as crianças foram integrando as rotinas diárias impostas pelos horários escolares no seu dia-a-dia, começaram a estruturar o seu padrão de sono, o que se reflectiu numa diminuição, ao longo do ano lectivo, dos problemas de sono. É de ressalvar ainda que, subjacente a esta alteração do índice de perturbação do sono, está a diminuição das queixas sintomáticas. Assim, ao longo do ano lectivo a sintomatologia do sono diminui, incidindo essa diminuição principalmente no nível da sonolência diurna. Porém, a sonolência ao acordar assume-se como uma excepção, na medida em que a sua prevalência aumenta ao longo desse período.

Quanto aos resultados encontrados no CDI, o índice de $6,2 \%$ do total da amostra com escores significativos para a depressão é semelhante aos índices apontados pela literatura (Bahls, 2002), que variam entre 
$4 \%$ e $10 \%$ em crianças e adolescentes. Esta percentagem diminui no segundo momento (4\%), havendo assim uma diminuição da sintomatologia depressiva ao longo do ano lectivo. A prevalência encontrada pode ser considerada expressiva, uma vez que indica que, de facto, a depressão está presente na infância. Importa salientar ainda que não se encontram diferenças estatisticamente significativas em relação ao género, idade, nível sócio-económico e rendimento académico. Desse modo, os resultados no nível das diferenças de género não foram ao encontro da literatura encontrada (Weissman \& Klerman, 1977), que sugere que, nas crianças, o sexo feminino tem maior tendência para a depressão que o sexo masculino, o que pode ser explicado pelo facto de as raparigas apresentarem uma maior predisposição para problemas de internalização do que os rapazes (Zahn-Waxler, Klimes-Dougan \& Slattery, 2000).

Relativamente à relação entre o sono e a depressão na infância, os resultados sugerem uma relação estatisticamente significativa entre a perturbação de sono e a sintomatologia depressiva, que se mantém ao longo do ano escolar. Esses dados corroboram estudos recentes evidenciando uma forte relação entre distúrbios de sono e distúrbios de humor (Ford \& Cooper-Patrick, 2001; Ivanenko e cols., 2005).

De facto, uma pobre qualidade de sono associa-se a queixas de humor, como a fadiga, ansiedade, irritabilidade e humor depressivo, bem como a défices no nível do funcionamento, como o decréscimo de atenção, motivação e eficiência (Gomes, Tavares \& Azevedo, 2005). Desse modo, essa conjectura permite compreender a relação negativa obtida neste estudo entre o rendimento académico e a sintomatologia depressiva e a qualidade do sono. Ao longo do ano lectivo houve uma diminuição da sintomatologia do sono e da depressão, o que possivelmente se reflectiu num aumento do desempenho escolar das crianças.

Posto isso, e tendo presente o objectivo do estudo - averiguar a prevalência e a relação entre os problemas de sono e a depressão em crianças em idade escolar - é possível concluir a presença desses dois quadros nosológicos nessa faixa etária, bem como uma associação entre ambos. Porém, esta associação é de pequena amplitude, pelo que se infere que possivelmente outros factores estejam a contribuir para a emergência e manutenção desta sintomatologia neste grupo etário.

Neste sentido, seriam úteis próximos estudos averiguarem a relação que une essas duas variáveis. Essa análise deveria incidir sobre os factores de risco e de protecção, de forma a termos uma noção mais clara dos determinantes da relação. Para tal, importa as próximas investigações terem em conta algumas limitações do estudo em questão. A metodologia adoptada neste estudo cingiu-se aos auto-relatos das crianças, as quais têm um conhecimento restrito e básico acerca do seu sono (Sadeh e cols., 2000), pelo que se constituiu numa limitação. Neste sentido, as futuras investigações deverão integrar outros instrumentos de avaliação para garantir uma maior fiabilidade dos resultados. Assim, seria vantajoso utilizar o auto-relato seguido de uma entrevista clínica orientada por psicólogos devidamente treinados, de forma a obter um conhecimento mais fidedigno do padrão de sono das crianças. Por outro lado, o facto de o estudo ter se desenvolvido em apenas dois momentos de avaliação, tornou-se igualmente uma limitação, pois não foi possível obter dados conclusivos acerca da relação entre as duas patologias. Desse modo, seria útil que os participantes fossem monitorizados ao longo do ano lectivo e não só em dois momentos, para que assim se possa ter uma perspectiva desenvolvimental que nos permita tirar ilações da relação que une os problemas de sono e a depressão infantil.

Por tudo isso, torna-se evidente a importância de desenvolver estudos nesse grupo etário, já que é na idade escolar que surgem as primeiras manifestações emocionais e comportamentais, que muitas vezes se associam aos problemas de sono. Porém, e graças à comorbilidade dos problemas de sono com outras perturbações, muitas vezes não são diagnosticados no contexto médico em detrimento de outras patologias. Esta situação pode, de certo modo, explicar a pouca sensibilidade parental para estas questões, já que não estão familiarizados com os problemas de sono, o que, por conseguinte, se reflecte numa higiene de sono inadequada. Assim, e tendo por base as implicações destes problemas no funcionamento psicossocial das crianças, seria útil que próximas investigações ajudassem a compreender os determinantes da relação entre o sono e a depressão infantil, para termos uma noção mais clara da necessidade de intervir nesta fase desenvolvimental, já que a intervenção precoce na idade escolar assume contornos importantes no desenvolvimento subsequente das crianças, constituindose numa oportunidade para promover a saúde, a performance escolar e a qualidade de vida das nossas crianças.

\section{Referências}

Aglio, D. D. \& Hutz, S. C. (2004). Depressão e desempenho escolar em crianças e adolescentes institucionalizados. Psicologia: Reflexão e Crítica, 17(3), 351-357.

American Psychiatric Association - APA (2000). DSM IV-TR - Manual de estatística e diagnóstico das perturbacões mentais. Lisboa: Climepsi.

Archbold, K. H., Pituch, K. J., Panahi. P. \& Chervin, R. D. (2002). Symptoms of sleep disturbances among children 
at two general paediatrics clinics. Journal of Pediatrics, 140, 97-102.

Bahls, S. C. (2002). Epidemiology of depressive symptoms in adolescents of a public school in Curitiba, Brasil. Revista Brasileira de Psiquiatria, 24, 63-67.

Blader, J. C., Koplewics, H. S., Abikoff, H. \& Foley, E. (1997). Sleep problems of elementary school children: A community survey. Archives of Paediatrics \& Adolescent Medicine, 151, 473-80.

Busse, R. S. \& Baldini, M. S. (1994). Distúrbios de sono em crianças. Pediatria, 16(4), 161-166.

Carskadon, M. A. (1990). Patterns of sleep and sleepiness in adolescents. Pediatrician, 17(1), 5-12.

Cassidy, J. (1994). The development of emotion regulation. Biological and Behavioural Considerations, 59(2), 228-249.

Choquet, M. \& Menke, H. (1987). Development of selfperceived risk behaviour and psychosomatic symptoms in adolescents: A longitudinal approach. Journal of Adolescence, 10(3), 291-308.

Clerget, S. (2001). Não estejas triste meu filho: compreender e tratar a depressão durante os primeiros anos de vida. Porto: Âmbar.

Compas, B. E., Eye, S. \& Grant, K. (1993). Taxonomy, assessment, and diagnosis of depression during adolescence. Psychological Bulletin, 114, 323-344.

Dahl R. E. (1996). The impact of inadequate sleep on children's daytime cognitive function. Seminaries of Paediatric Neurology, 3, 44-50.

Diest, R. van (1990). Subjective sleep characteristics as coronary risk factors, their association with type A behaviour and vital exhaustion. Journal of Psychosomatic Researcher, 34(4), 415-426.

Ford, D. E. \& Cooper-Patrick, L. (2001). Sleep disturbances and mood disorders: an epidemiologic perspective. Depression and Anxiety, 14, 3-6.

Gau, S. F. \& Soong, W. T. (1995). Sleep problems in junior high school students in Taipei. Sleep, 18, 667-673.

Gillin, J. C. (1998). Are sleep disturbances risk factors for anxiety, depressive and addictive disorders? Acta Psychiatrica Scandinavica, suplement, 393, 39-43.

Gomes, A. A., Tavares, J. \& Azevedo, M. H. P. (2005). Daytime correlates of poor sleep in undergraduates during final exams. Journal of Sleep Research, 13 (Suppl. 1), 82-93.

Gonçalves, M. \& Dias, P. (1999). Avaliação da ansiedade e da depressão em crianças e adolescentes (STAIC-C2; CMAS-R, FSSC-R e CDI): estudo normativo para a população portuguesa. Em A. P. Soares, S. Araújo \& S.
Caíres (Orgs.). Avaliação psicológica: formas e contextos (pp. 553-564). Braga: APPORT.

Gozal, D. (1998). Sleep-disordered breathing and school performance in children. Paediatrics, 3(102), 616-620.

Harrington, R., Kerfoot, M. \& Verduyn, C. (1999). Developing needs led child and adolescent mental health services: issues and prospects. European Child and Adolescent Psychiatry, 8, 1-10.

Ivanenko, A., Cabtree, M. V. \& Gozal, D. (2005). Sleep and depression in children and adolescents. Sleep Medicine Reviews, 9, 115-129.

Kahn, A., Van de Merct, C., Rebuffat, E., Mezin. M. J., Sotiaux, M., Blum, D. \& Hennart, P. (1989). Sleep problems in healthy preadolescents. Paediatrics, 84, 542-546.

Klein, J., Fernandes, C. \& Gonçalves, A. (2005a). Assessing sleep-wake complaints in school-aged children: Portuguese properties and norms of the SWEL. Trabalho apresentado no $1^{\circ}$ Congresso Nacional de Psicologia da Saúde e Medicina Comportamental. Universidade do Minho, Braga, Portugal.

Klein, J., Fernandes, C. \& Gonçalves, A. (2005b). Epidemiological study of sleep disorders in school-aged childrens of portuguese schools. Psychophysiology, 42(Supplement 1), 74.

Kovacs M (1985) The Children's Depression Inventory (CDI). Psychopharmacology Bulletin, 21, 995-998.

Liu, X., Sun, Z., Uchyiama, M., Shibui, K., Kim, K. \& Okawa, M. (2000). Prevalence e correlates of self report sleep problems in Chinese schoolchildren. Sleep, 23, 1053-1062.

Madeira, I. R. \& Aquino, L. A. (2003). Problemas de abordagem difícil: "não come" e "não dorme". Jornal de Pediatria, supl. 1, 43-54.

Mericangaas, K. R. \& Angst, J. (1995). The challenge of depressive disorders in adolescence. Em Rutter, M. Psychosocial disturbances in young people (pp. 3-6). Londres: Cambridge University Press.

Moran, G. \& Pederson, D. R. (1998). Proneness to distress and ambivalent relationships observed in the home. Infant Behaviour and Development, 21, 493-503.

Morrel, J. \& Steele, H (2003). The role of attachment security, temperament, maternal perception, and caregiving behaviour in persistent infant sleeping problems. Infant Mental Health Journal, 24(5), 447-468.

Rehm, L. P. \& Sharp, R. N. (1996). Estratégias para a depressão infantil. Em M. Reinecke, F. Dattilio \& A. Freeman (Orgs.). Terapia cognitiva com crianças e adolescentes (pp. 91-104). São Paulo: Artes Médicas. 
Riemann, D., Berger, B. \& Voderholzer, U. (2001). Sleep and depression - results from psychobiological studies: An overview. Biological Psychology, 57, 67-103.

Roberts, R. E., Roberts, R. C. \& Chen, G. I. (2001). Functioning of adolescents with symptoms of disturbed sleep. Journal of Youth and Adolescence, 30, 1-17.

Rona, K. J., Li, L., Guilford, M. C. \& Chinun, S. (1998). Disturbed sleep: Effects of socio-cultural factors and illness. Archives of Disease in Childhood, 78, 28-51.

Rudolph, K., Hammen, C. \& Burge, D. (1997). A cognitive-interpersonal approach to depressive symptoms in preadolescent children. Journal of Abnormal Child Psychology, 25(1), 33-45.

Sadeh, A. \& Anders, T. F. (1993). Infant sleep problems: Origins, assessment and intervention. Infant Mental Health Journal, 14, 17-34.

Sadeh, A., Raviv, A. \& Gruber, R. (2000). Sleep patterns and sleep disruptions in school-age children. Developmental Psychology, 36(3), 291-301.

Spitz, R. (1965). The first year of life. New York: International Universities Press.

Stein, M. A., Mendelsohn, J., Obermeyer, W. H., Amromin, J. \& Benca, R. (2001). Sleep and behavior problems in school-aged children. Paediatrics, 107(4), 1-9.

Steinberg, L. (1999). Adolescence. Boston: McGraw-Hill.

Stores, G. (2001). Normal sleep including developmental aspects. Em G. Stores \& L. Wiggs (Orgs.). Sleep disturbance in children and adolescents with disorders of development; its significance and management (pp.
10-14). Clinics in Developmental Medicine (vol. 155). Cambridge: University Press.

Stores, G. (2003). Misdiagnosing sleep disorders as primary psychiatric conditions. Advances in Psychiatric Treatment, 9, 69-77.

Strecht, P. (1997). Crescer no vazio (2 $2^{\mathrm{a}}$ ed.). Lisboa: Assírio \& Alvim.

Szuba, P. M. (2001). The psychobiology of sleep and major depression. Depression and Anxiety, 14, 1-2.

Weissman, M. M. \& Klerman, G. L. (1977). Sex differences and the epidemiology of depression. Archives of General Psychiatry, 34, 98-111.

Weissman, M. M., Greenwald, S., Nino-Murcia, G. \& Dement, W. C. (1997). The mobility of insomnia uncomplicated by psychiatric disorders. General Hospital Psychiatry, 19, 245-250.

Zahn-Waxler, C., Klimes-Dougan, B. \& Slattery, M. J. (2000). Internalizing problems of childhood and adolescence: Prospects, pitfalls, and progress in understanding the development of anxiety and depression. Development and Psychopathology, 12, 443-466.

Zavaschi, M. L. S, Satler, F., Poester, D. Vargas, C. F., Piazenski, R., Rohde, L. A. P. \& Eizirik, C. L. (2002). Associação entre trauma por perda na infância e depressão na vida adulta. Revista Brasileira de Psiquiatria, 24, 189-195.

Aprovado em agosto de 2006 Reformulado em abril de 2007 Aprovado em abril de 2007

Sobre os autores:

Filipa Serrão é licenciada em Psicologia pela Universidade do Minho, onde desenvolveu projetos no âmbito das perturbações de sono. Atualmente, exerce funções de psicóloga clínica na Direção Regional de Educação Especial e Reabilitação e na Policlínica de Câmara de Lobos, na Região Autônoma da Madeira. Tem uma vasta experiência clínica na área das problemáticas escolares e clínicas das crianças.

John M. Klein é doutor em Psicologia Clínica e atualmente é professor na Universidade de Trier (Alemanha). Tem publicado e apresentado vários trabalhos nas seguintes áreas de interesse: Psicopatologia, Processos e Resultados Terapêuticos, Avaliação Psicológica e Neurociências.

Alda Gonçalves é licenciada em Psicologia pela Universidade do Minho, onde atualmente completa o mestrado em Psicologia Clínica. Nos últimos anos, tem-se dedicado a estudar as perturbações do comportamento alimentar e as perturbações de sono. 